\title{
Mechanical characterization of precipitation hardened Al7075-grey cast iron powder reinforced metal matrix composites
}

\author{
Jamaluddin Hindi ${ }^{1^{*}}$, Kini Achuta ${ }^{1}$ and Murthy Amar ${ }^{1}$ \\ ${ }^{1}$ Department of Mechanical and Manufacturing Engineering, Manipal Institute of Technology, \\ Manipal Academy of Higher Education, 576104, India
}

\begin{abstract}
A17075 alloy is the most commonly used by the aerospace industry. A17075 alloy is characterized by its improved properties such as higher toughness, specific strength and hardness. The current work focuses on the preparation and characterization of age hardened Al7075-Grey cast iron composites. Two stage stir casting technique is used for the preparation of the composite. Age hardening treatment is imparted to enhance the mechanical characteristics. The variation of hardness and tensile strength with respect to aging temperature and percentage of reinforcement is analyzed. The composites exhibit higher hardness and tensile strength as the reinforcement percentage is increased at an aging temperature of $100^{\circ} \mathrm{C}$.
\end{abstract}

\section{Introduction}

Composite material is a combination of dissimilar materials which result in property enhancement than those of the individual elements. There will not be any change in the chemical, physical, and mechanical properties of individual elements i.e. matrix and reinforcement [1-4]. Composite materials are characterized by high strength to weight ratio, higher stiffness with low density resulting in considerable reduction in the weight of the component. Enhancement in the desirable properties is mainly due to the reinforcement which is generally harder, refractory, and stiffer than the base material [5]. The reinforcement phase can be continuous or discontinuous fiber or particulate. Metal matrix composites can be defined as a combination of two or more dissimilar materials, one of which is a metal, in which enhancement of desirable properties are achieved by systematic combinations of different constituents [6-8]. Very high specific strength and specific modulus can be achieved in MMCs consisting of continuous or discontinuous fibers, whiskers, or particles [9].

\footnotetext{
* Corresponding author: jamalhindi@gmail.com
} 
Aluminum alloy composites are hardened by reinforcement of ceramic refractory particulates into the base matrix material. Due to their innate nature and creation of nucleation sites in the matrix material, reinforcements can enhance tensile related properties of the matrix material [10-12]. Particulate reinforced composites are characterized by economic manufacturing methods with finer grains. Selection of the type of reinforcement is based on the property enhancement and monetary investment [13,14]. Generally, continuous fiber reinforced MMCs have unidirectional properties in the direction of the fiber, but are costly. Chopped fibers can produce significant property enhancement in the two dimensionally in the direction of their orientation, at moderate cost. MMCs provide moderate but isotropic properties [15-19].

Grey cast iron is one of the types of cast irons which have unique microstructure containing carbon in free and combined form. Carbon is present as cementite and graphite. The weight percentage of these two constituents can be altered by the addition of graphite formers like $\mathrm{Si}$. The fractured surface of the grey cast iron specimen is greyish in colour due to the presence of free carbon as graphite and combined carbon as cementite. The graphite also provides gray cast iron with an additional damping capacity for the alloy or matrix since it absorbs the energy [21-23].

\section{Materials and methods}

\subsection{Al 7075 alloy}

Al7075 alloy is the most commonly used by the aerospace industry. Al7075 alloy is characterized by its improved properties such as higher toughness, specific strength and hardness. The Al-Zn-Mg alloys [A17075] are used in as cast and age hardened condition. As cast alloys are generally homogenized. Table 1 shows the chemical composition of A17075 alloy [20].

Table 1. Chemical composition of Al7075 alloy.

\begin{tabular}{|c|c|c|c|c|c|c|c|c|c|}
\hline $\mathrm{Al}$ & $\mathrm{Cr}$ & $\mathrm{Cu}$ & $\mathrm{Fe}$ & $\mathrm{Mg}$ & $\mathrm{Zn}$ & $\mathrm{Si}$ & $\mathrm{Mn}$ & $\mathrm{Ti}$ & Others \\
\hline 89.79 & 0.08 & 1.35 & 0.3 & 2.21 & 5.67 & 0.4 & 0.08 & 0.06 & 0.06 \\
\hline
\end{tabular}

\subsection{Grey cast iron}

The chemical composition of grey cast iron is shown in table 2 .

Table 2. Chemical composition of the grey and cast iron (wt.\%).

\begin{tabular}{|l|l|l|l|l|l|l|}
\hline $\begin{array}{l}\text { Element in } \\
\text { wt.\% }\end{array}$ & $\mathrm{C}$ & $\mathrm{S}$ & $\mathrm{P}$ & $\mathrm{Si}$ & $\mathrm{Mn}$ & $\mathrm{Fe}$ \\
\hline $\mathrm{GCI}$ & 3.61 & 0.024 & $\leq 0.022$ & 1.30 & 0.41 & $\mathrm{Bal}$ \\
\hline
\end{tabular}


Grey cast iron has good potential as a reinforcement material due to following reasons:

- It is also an industrial waste which comes in the form of chips during machining operation. These chips can be re casted to be used as reinforcement.

- Grey cast iron is hard due to the presence of cementite but also has high selflubricating property and machinability due to the presence of free graphite in the form of flakes.

- It serves as a hybrid reinforcement because microscopically there are three phases in $\mathrm{GCI}$ at room temperature i.e. cementite, ferrite and graphite.

- It will not react with A17075 which will be used as a base matrix material.

\subsection{Preparation of the reinforcement phases}

Grey cast iron (GCI) rod is cast using chill casting technique with secondary operation i.e. turning operation to machine the casting to remove foreign material inclusions near the mould wall and slag at the top of the casting. Hardness values at three sample zones of casting is measured using Rockwell B scale to analyse the homogeneity of hardness values of the sample. To achieve homogenous chemical composition and hardness, the specimen is annealed at $800^{\circ} \mathrm{C}$ for 10 hours [24-26] The casting is turned in lathe to collect grey cast iron dust (debris), washed and preheated to $300^{\circ} \mathrm{C}$ for 1 hour to remove volatile substances present. Debris is ground in planetary ball mill as shown in figure 2 to reduce the size of particles upto $50 \mu \mathrm{m}$ and sieving is performed to obtain uniform grade of particles. Sieving and grinding cycles is repeated till the required quantity of uniform grade of particles are obtained. The particles were seived through ASTM-140 sieve to obtain uniform grade of particles of average size $50 \mu \mathrm{m}$ [18].

\subsection{Stir casting of Al7075-GCl composites}

Al7075-T6 rods of 1 inch diameter and 7 inch length were procured from the supplier. It was cut to 1 inch length and required number of pieces were placed in the crucible and melted in a furnace at $750^{\circ} \mathrm{C}$. After complete melting hexa chloroethane was added as a degasification agent. Alkaline powder was added to remove the slag. Permanent mold cavities for cylindrical and flat billets are cleaned using a wire brush and are coated with graphite powder and water emulsion. Assembled molds were preheated to a temperature of $450^{\circ} \mathrm{C}$ for an hour in a separate furnace. Reinforcement prior to the addition to the molten metal was separately pre heated to $450^{\circ} \mathrm{C}$ to remove volatile substances [9]. Three compositions of the composite were prepared by varying the weight percentage of the reinforcement. Stirring of the molten metal was carried using a mechanical stirrer placed on top of the Induction furnace. Required amount of reinforcement was added to the melt through a funnel placed in position. Vortex was created by rotating the stirrer at suitable speed, two step stirring was carried out to overcome heat loss and drop in furnace temperature. After the completion of stirring the molten metal with reinforcements was poured to a pre heated permanent mold and allowed to solidify at room temperature.

\section{Results and discussion}

\subsection{Measurement of Peak hardness}

Peak hardness values are noted for all the compositions. Age hardening curves were plotted for each specimen at half an hour interval. From the analysis of age hardening curves it is evident that lower the temperature of aging, higher will be the peak hardness value. As the 
aging temperature is increased peak hardness can be obtained at a faster rate but sacrificing on the value of peak hardness. At both lower and higher aging temperature, material exhibits higher peak hardness as the weight percentage of the reinforcement is increased. Figure 1 and 2 shows Age hardening curve at lower and higher aging temperature at 100 and $200^{\circ} \mathrm{C}$. Figure 3 shows variation in peak hardness with respect to wt. $\%$ of GCI for ascast and age hardened specimens.

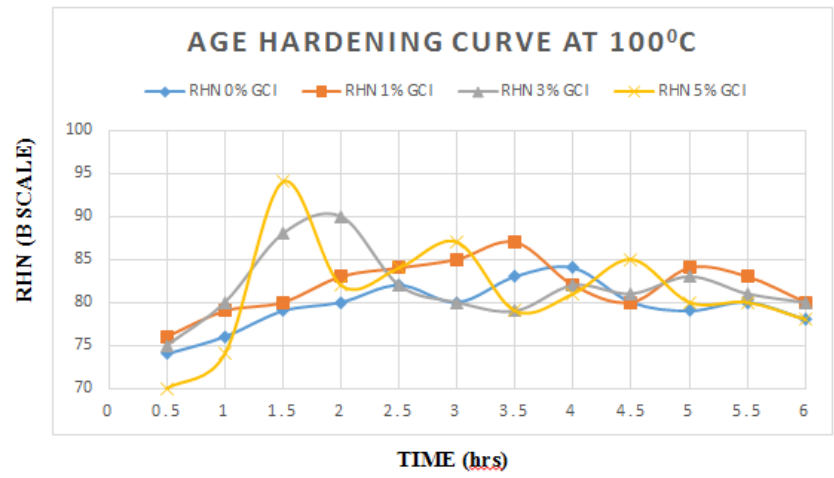

Fig. 1. Age hardening curve at lower aging temperature $100^{\circ} \mathrm{C}$.

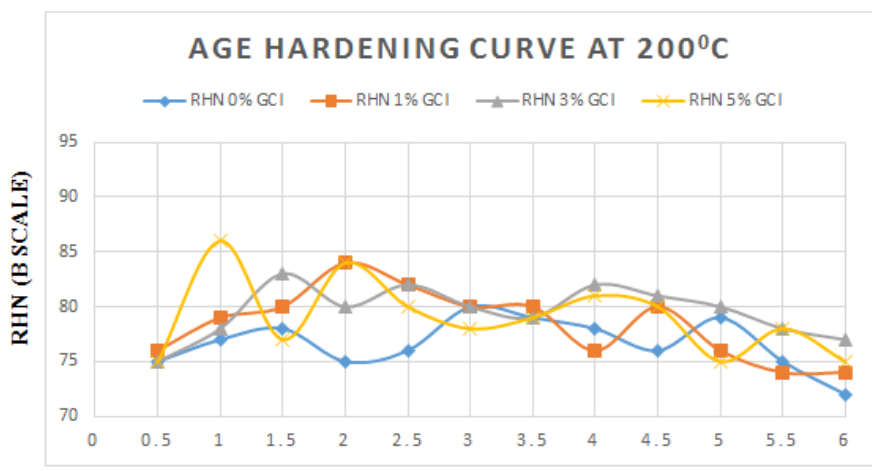

TIME (hrs)

Fig. 2. Age hardening curve at higher aging temperature $200^{\circ} \mathrm{C}$.

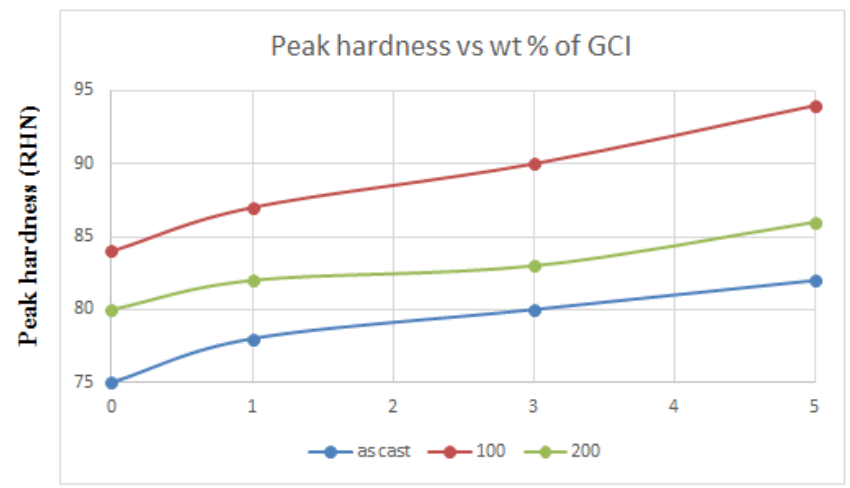

Wt $\%$ of GCI

Fig. 3. Peak hardness vs wt.\% of GCI for as-cast and age hardened specimens. 


\subsection{Measurement of tensile strength}

From the analysis of the graph shown in figure, it is evident that lower the temperature of aging, higher will be the ultimate tensile strength value. At both lower and higher aging temperature, material exhibits higher ultimate tensile strength as the weight percentage of the reinforcement is increased. Figure 4 shows the variation in ultimate tensile strength with respect to wt.\% of GCI for as-cast and age hardened specimens. The ductility decreases as the percentage of reinforcement is increased irrespective of the aging temperature as shown in figure 5 .

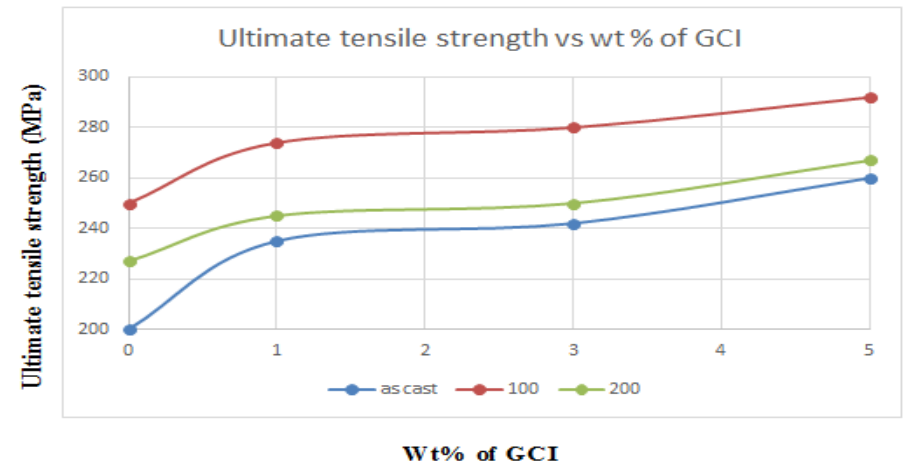

Fig. 4. Ultimate tensile strength vs wt. $\%$ of GCI for as-cast and age hardened specimens.

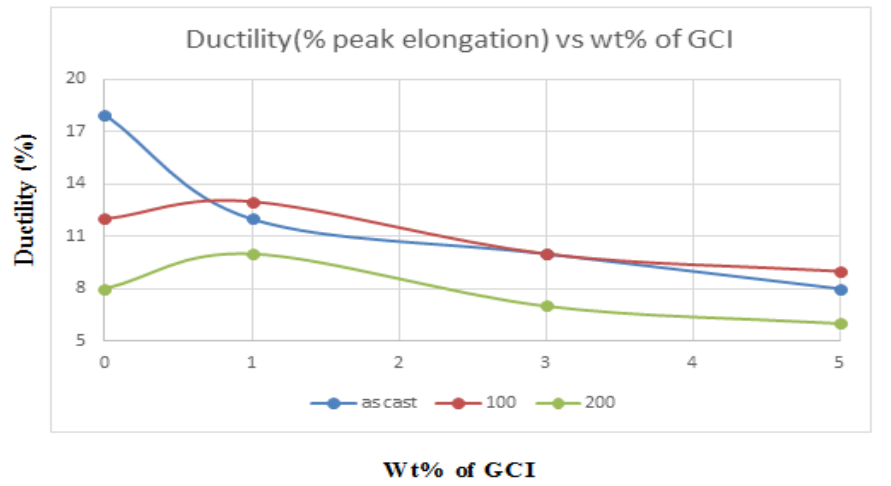

Fig. 5. Ductility vs wt.\% of GCI for as-cast and age hardened specimens.

\subsection{Microstructure analysis}

The specimens are systematically polished with a series of silicon carbide embedded emery papers starting from coarser 100 microns to finer 600 microns in the steps of 100 microns. At every stage of polishing specimens are water washed and dried with acetone. Super finishing operation known as buffing is performed on disc polisher with wet diamond paste of 50 microns. Finally, the mirror like polished specimens is etched with etchant (Keller's reagent) [6]. Microstructures of all the samples are recorded in metallurgical microscope at $300 \mathrm{X}$ magnification. Figure 6 shows the microstructure of heat treated composites at 300X as recorded by ImageAnalyzer. Homogenised specimens show better dispersion of reinforcement without clustering. 


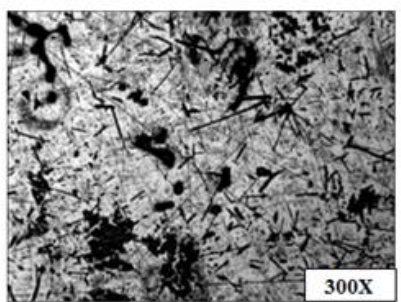

(a)

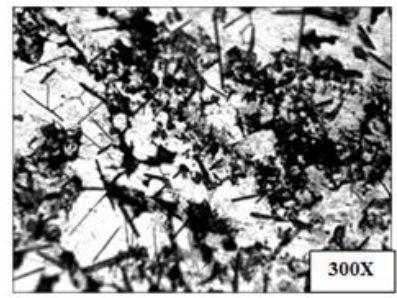

(b)

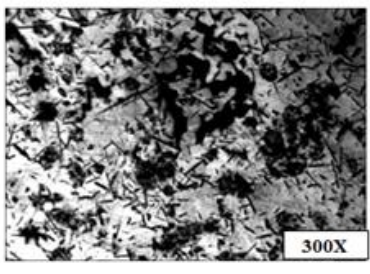

(c)

Fig. 6. Microstructure of homogenized (a) AA7075 composite with 1\% Grey cast iron powder (b) AA7075composite with 3\% Grey cast iron powder (c) AA7075 composite with 5\% Grey cast iron powder.

\section{Conclusions}

Al 7075 alloy can be successfully reinforced with grey cast iron powder using two step stir casting technique. Age hardening treatment significantly improves the mechanical properties of the composite material. Lower the aging temperature better is the peak hardness values in age hardening. Substantial improvement in the bulk hardness is observed in the composite with increase in the weight percentage of reinforcement. Substantial improvement in the ultimate tensile strength is observed in the composite with increase in the weight percentage of reinforcement. Peak hardness and maximum tensile strength was observed in $\mathrm{Al} 7075-5 \% \mathrm{GCI}$ aged at $100^{\circ} \mathrm{C}$. The ductility decreases as the percentage of reinforcement is increased. The microstructure study reveals uniformity in the reinforcement distribution without any agglomeration.

\section{References}

1. Akhlaghi and Zare-Bidaki, "Influence of graphite content on the dry sliding and oil impregnated sliding wear behavior of Al2024-graphite composites produced by in situ powder metallurgy method", Wear, 266, 2009, 37-45.

2. Ali Kalkanli and Sencer Y1lmaz, "Synthesis and characterization of aluminum alloy 7075 reinforced with silicon carbide particulates", Materials and Design, 29, 2008, 775-780.

3. Anand Pai, Sathya Shankar Sharma, Raynor Errol D'Silva and Nikhil, "Effect of graphite and granite dust particulates as micro-fillers on tribological performance of Al 6061-T6 hybrid composites", Tribology International, 92, 2015, 462-471.

4. Baradeswaran A and Elaya Perumal, "Study on mechanical and wear properties of Al 7075/A12O3/graphite hybrid composites”, Composites, 56, 2014, 464-471.

5. Baradeswaran A, Vettivel, Elaya Perumal, Selvakumar and Franklin Issac, "Experimental investigation on mechanical behaviour, modeling and optimization of wear parameters of B4C and graphite reinforced aluminium hybrid composites", Materials and Design, 63, 2014, 620-632.

6. Bayazida S.M, Farhangia, Asgharzadehb and Radanc "Effect of cyclic solution treatment on microstructure and mechanical properties of friction stir welded $7075 \mathrm{Al}$ alloy”, Materials Science and Engineering, 649, 2014, 293-300.

7. Campbell F.C, "Metal matrix composites" ASM international, 2010, 538-539. 
8. Clark. R, Coughran, Traina, Hernandez. A and Scheck, "On the correlation of mechanical and physical properties of 7075-T6 Al alloy", Engineering Failure Analysis, 12, 2005, 520-526.

9. Deaquino Lar, Soltani, Bahrami, Gutiérrez Castañeda, and García-Sánchez, "Tribological characterization of Al7075-graphite composites fabricated by mechanical alloying and hot extrusion”, Materials and Design, 67, 2015, 224-231.

10. Jin-feng, Zhuo-wei peng, Chao-xing li, and Zhi-qiang Jia, "Mechanical properties, corrosion behavior and microstructures of 7075 aluminium alloy with various aging treatments", Transactions of Nonferrous Metals Society of China, 18, 2008, 755-762.

11. Kiran T.S, Prasanna Kumar, Basavarajappa and Viswanatha, "Dry sliding wear behavior of heat treated hybrid metal matrix composite using Taguchi techniques", Materials and Design, 63, 2014, 294-304.

12. Mahathaninwonga N T, Plookphola, J, Wannasina, and Wisutmeth angoonb, "T6 heat treatment of rheocasting 7075 Al alloy", Materials Science and Engineering, 532, 2012, 91-99.

13. Michael Oluwatosin Bodunrin, Kenneth Kanayo, and Alaneme Lesley Heath Chown "Aluminium matrix hybrid composites: a review of reinforcement philosophies; mechanical, corrosion and tribological characteristics", Journal of Materials Research and Technology, 169, 2015, 169-181.

14. Murat Baydogan, Hüseyin Çimenoglu and Sabri Kayalı, "Study on sliding wear of a 7075 aluminum alloy", Wear, 257, 2004, 52-61.

15. Nikhilesh Chawla and Yu-Lin Shen, "Mechanical behavior of particle reinforced metal matrix composites", Advanced Engineering Materials, 3, 2001, 357-370.

16. Ravinder Kumar and Suresh Dhiman, "A study of sliding wear behaviors of Al7075 alloy and A17075 hybrid composite by response surface methodology analysis", Materials and Design, 50, 2013, 351-359.

17. Reda Y, Abdel-Karim, and Elmahallawi, "Improvements in mechanical and stress corrosion cracking properties in Al7075 via retrogression and re aging", Materials Science and Engineering, 485, 2008, 468-475.

18. Rupa Dasgupta, "The stretch, limit and path forward for particle reinforced metal matrix composites of 7075 Al-alloys" Scientific Research Engineering, 2, 2010, 237 256.

19. Sahin $\mathrm{Y}$, "Preparation and some properties of $\mathrm{SiC}$ particle reinforced aluminium alloy composites", Materials and Design, 24, 2003, 671-679.

20. Satish Kumar Thandalama, Subramanian Ramanathana and Shalini Sundarrajan, "Synthesis, microstructural and mechanical properties of ex situ zircon particles ( $\mathrm{ZrSiO} 4)$ reinforced metal matrix composites (MMCs): a review", Journal of Materials Research and Technology, 4, 2015, 333-347.

21. Siavash gholami, Esmaeil emadoddin, Mohammad tajally and Ehsan borhani, "Friction stir processing of $7075 \mathrm{Al}$ alloy and subsequent aging treatment" Transactions of Nonferrous Metals Society of China, 25, 2015, 2847-2855.

22. Uvaraja V.C and Natarajan, "Tribological behavior of heat treated A17075 aluminium metal matrix composites", Indian Journal of Engineering and Material Sciences, 22, 2015, 51-61.

23. Veeresh Kumar G.B, Rao, Selvaraj, and Bhagyashekar, "Studies on Al6061-SiC and Al7075-A12O3 metal matrix composites", Journal of Minerals and Materials Characterization Engineering, 9, 2010, 43-55. 
24. Warren H, Hunt, Darrell and Herling Text book, "Aluminium metal matrix composites", Advanced Materials and Processes, 2004.

25. Yoshiro Iwai, Hidetomo.Yonede, and Tomomi.Honda, "Sliding wear behaviour of SiC whisker reinforced aluminum composite", Wear, 181, 1995, 594-602.

26. Zeynep Taşliçukur, Gözde S.altuğ and Şeyda polat, "Characterization of microstructure and fracture behavior of GG20 and GG25 cast iron materials used in valves", Metals, 5, 2012, 23-25.

27. Zhang, Long S, and Flower, "Light alloy composite production by liquid metal infiltration", Composites, 25, 1994, 380-392. 